\title{
The Relationship between PI-RADS Categories and Incidental Findings in Multiparametric Prostate MRI
}

\author{
Suna Sahin Ediz ${ }^{1}$ and Nesrin Gunduz ${ }^{2}$ \\ ${ }^{1}$ Department of Radiology, University of Health Sciences, Kartal Dr. Lutfi Kirdar City Hospital, Istanbul, Turkey \\ ${ }^{2}$ Department of Radiology, Faculty of Medicine, Medeniyet University, Goztepe Education and Research Hospital, Istanbul, Turkey
}

\begin{abstract}
Objective: To find the frequency and significance of extraprostatic incidental findings (ep-IFs) during multiparametric-magnetic prostate resonance imaging ( $\mathrm{mp}-\mathrm{MRI})$, and compare them with prostate imaging reporting and data system (PI-RADS) outcomes.

Study Design: Analytical study.

Place \& Duration of Study: Goztepe Education and Research Hospital, Istanbul, Turkey, from June 2019 to January 2020.

Methodology: Images of 185 men, who underwent mp-MRI, were reviewed and ep-IFs were also classified as urologic or nonurologic and benign or malign. The PI-RADS score was also recorded in biopsy-naïve subjects or in whom a sufficient time (which would not impair imaging) was elapsed after the biopsy. The cases were also divided into two groups, according to the PI-RADS score (Group 1: PI-RADS 1 or 2, Group 2: PI-RADS 3 or more) and the incidental findings (IFs) were compared between the groups.

Results: Overall, 139 ep-IFs were detected in 88 (47.6\%) patients. The remaining 97 (52.4\%) cases were free of ep-IFs. The ep-IFs were benign in $85(96.6 \%)$ and malignant in $3(3.4 \%)$ cases. The frequency of total ep-IFs did not differ between groups 1 and 2 (47.8\% vs. $47.6 \%$, respectively, $p>0.05)$.

Conclusion: Extra prostatic incitental findings are frequently encountered during mp-MRI, benign ep-IFs are quite frequent; although rare malignant ep-IFs may be subject to being missed due to focused analysis and interpretation of prostate. PI-RADS scoring system does not contribute to the diagnosis of incidental mp-MRI.
\end{abstract}

Key Words: Incidental findings, Multiparametric prostate MRI, Prostate cancer, MP-MRI.

How to cite this article: Ediz SS, Gunduz N. The Relationship between PI-RADS Categories and Incidental Findings in Multiparametric Prostate MRI. J Coll Physicians Surg Pak 2021; 31(09):1030-1034.

\section{INTRODUCTION}

Prostate cancer (PCa) is, apart from hereditary cases, the most common cancer in advanced age males. ${ }^{1}$ It is the third most common cause of cancer-related mortality among men in the developed world. ${ }^{2}$ The causes and risk factors of prostate cancer are related to the age, endogenous hormone balance, genetic (predisposing) factors, and environmental factors, including a fatty diet. ${ }^{3}$

The natural onset of PCa is not completely elucidated, but the disease progression seems to be determined by the stage and the grade of the tumor. After widespread adoption of PSA testing, prostate cancer rate increased followed by a subsequent decline and recent stabilisation. ${ }^{4}$

Correspondence to: Suna Sahin Ediz, Department of Radiology, University of Health Sciences, Kartal Dr. Lutfi Kirdar City Hospital, Istanbul, Turkey

E-mail: suna-sahin@hotmail.com

Received: February 01, 2021; Revised: July 29, 2021;

Accepted: August 04, 2021

DOI: https://doi.org/10.29271/jcpsp.2021.09.1030
The 5-year survival rate for most men with local or regional PCa is nearly $100 \%$; and for advanced Pca, 3-year, 5-year and 7year overall survival rates are $79.36 \%, 61.46 \%$ and $49.15 \%$, respectively. ${ }^{5,6}$

About imaging, visualisation of PCa has always been difficult due to heterogenous complexity of PCa, multifocality and deep location of tissue. ${ }^{7}$ Digital rectal examination (DRE), serum PSA (prostate-specific antigen) levels, and histopathologic evaluation of prostate biopsies are valuable parameters to the diagnosis of PCa. MR-targeted biopsy is a popular diagnostic method with more certain and smarter features for PCa. It has been shown to detect more high-risk cases, while simultaneously decreasing the detection of low-risk, non-significant $\mathrm{PCa} .{ }^{8,9}$

$\mathrm{mp}-\mathrm{MRI}$ of the prostate is now widely used to improve prostate cancer detection, localisation, and staging. ${ }^{10}$ Besides the prostate gland, the possible pathologies in the field of view, such as abdominal and pelvic organs, vasculature and bones, can also be detected on $\mathrm{mp}$-MRI. This has the contribution for management of PCa staging through evaluation of the seminal vesicles, bones, and regional pelvic lymph nodes for potential PCa involvement. $^{5}$ 
$\mathrm{mp}-\mathrm{MRI}$ provides reliable information regarding the diagnosis, staging, and monitoring of therapy in patients with PCa. The detection of IFs not always related to the primary purpose of the examination increased because of the widespread utilisation of mp-MRI. ${ }^{11-14}$

However, as in all imaging techniques, incidental findings (IFs) during $\mathrm{mp}$-MRI require identification. The frequency and significance of IFs during mp-MRI were not well studied. There are two main studies on this subject in the literature. ${ }^{11,15}$ In previous studies, the presence of IFs was evaluated according to the risk group and age groups of the patients. The most important benefit of using mp-MRI in the diagnosis of prostate cancer is to give prostate imaging reporting and data system (PI-RADS) score related to lesions. A study comparing the frequency and significance of IFs according to PI-RADS groups has not been published yet. This study aimed to find the frequency and significance of extraprostatic incidental findings (ep-IFs) during mpMRI, and compare them with PI-RADS outcomes.

\section{METHODOLOGY}

This analytical study was approved by the local Ethics Committee and conducted retrospectively according to the principles of the World Medical Association Declaration of Helsinki's Ethical Principles for Medical Research Involving Human Subjects.

The images of 185 men, who underwent mp-MRI from June 2018 to January 2019 were reviewed from picture archiving and communication system (PACS). Patients, who underwent $\mathrm{mp}$ $M R I$ due to the presence of significant DRE findings for PCa and/ortPSA elevation, were included in the study.

A 1.5 TMRI scan system (GE750, GE Healthcare) with an abdominal eight-channel surface phased array coil was used to perform the imaging. Axial, sagittal, and coronal T2-weighted sequence (TR/TE, 4137/86 ms), DWI (TR/TE, 4200/90 ms) images with a maximum b value of $1400 \mathrm{~s} / \mathrm{mm} 2$ and corresponding ADC maps were obtained for analysis, section thickness: $3 \mathrm{~mm}$. DCE images were obtained after intravenous injection of gadopentetate dimeglumine at a dose of $0.1 \mathrm{mmol} / \mathrm{Kg}$ of body weight and a rate of $3 \mathrm{~mL} / \mathrm{sec}$ by using an automatic injector.

One MRI specialised body radiologist reviewed all MRIs from the PACS and PI-RADS scores and all extraprostatic findings were recorded. An incidental finding was defined as an incidentally discovered mass or lesion, detected by MRI performed for prostate cancersuspicion.

IFs were classified as urologic or non-urologic and benign or malign. The PI-RADS score was also recorded in biopsy-naïve subjects or in whom a sufficient time (which would not impair imaging) was elapsed after the biopsy. The cases were also divided into two groups, according to the PI-RADS score (Group 1: PI-RADS 1 or 2, Group 2: PI-RADS 3 or more) and the IFs were compared between the groups.

Statistical analysis was conducted by using SPSS software (ver- sion 20.0. Armonk, NY, USA: IBM Corp). Shapiro-Wilk test was used to assess the normal distribution of data. Continuous variables were expressed as median (the interquartile range: IQR) and categorical variables are expressed as numbers and percentages. Chi-square test or Fisher's Exact test was used to assess the categorical data. Statistical significance level was set at $p$ $<0.05$.

\section{RESULTS}

The average age of the patients was 64 years (IQR: 58-69.5). Overall 139 ep-IFs were detected in 88 (47.6\%) patients. The remaining $97(52.4 \%)$ cases were free of ep-IFs. Overall, 48 (25.9\%) patients had only one IF, 29 (15.7\%) patients had two Ifs, and 11 (5.9\%) patients had three IFs. The ep-IFs were benign in $85(96.6 \%)$ and malignant in $3(3.4 \%)$ cases. The malignant ep-IFs included two bladder cancer and one rectal neuroendocrinetumor (Figures 1 and 2 ). Which were not previously diagnosed, benign ep-IF $(n=136)$ included $9(6.6 \%)$ Tarlov cysts, 4 (2.9\%) lymphadenopathies, $3(2.2 \%)$ intraperitoneal free fluid, $26(19.1 \%)$ hydrocele, 30 (22.1\%) bladder abnormalities (increasedbladder wall thickening, trabeculation, diverticules, calcule), 13 (9.6\%) sigmoid diverticules, 51 (37.5\%) inguinal hernias (Figure 3).

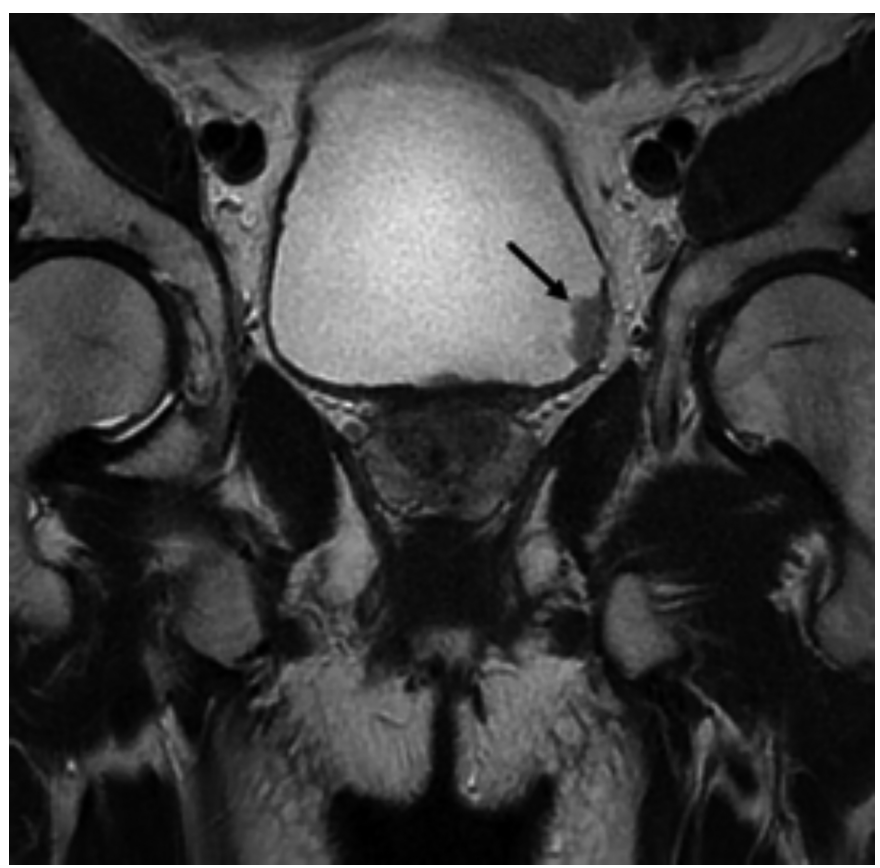

Figure 1: T2W coronal section of a patient, who underwent multiparametric prostate MRI, shows an incidental bladder mass (arrow) which was subsequently proved to be uroepithelial carcinoma.

PI-RADS scores were reported in 170 (91.9\%) of 185 patients. The distribution of patients for PI-RADS scores 1 to 5 in 170 patients were $1(0.6 \%), 45(26.5 \%), 73(42.9 \%), 26(15.3 \%)$ and $25(14.7 \%)$ cases, respectively.

The frequency of total ep-IFs did not differ between PI-RADS groups 1 and 2 (47.8\% vs. 47.6\%, respectively, p:0.118). The individual frequency of every single benign ep-IF also did not differ between the two groups ( $p>0.05$ for all, Tablel). 


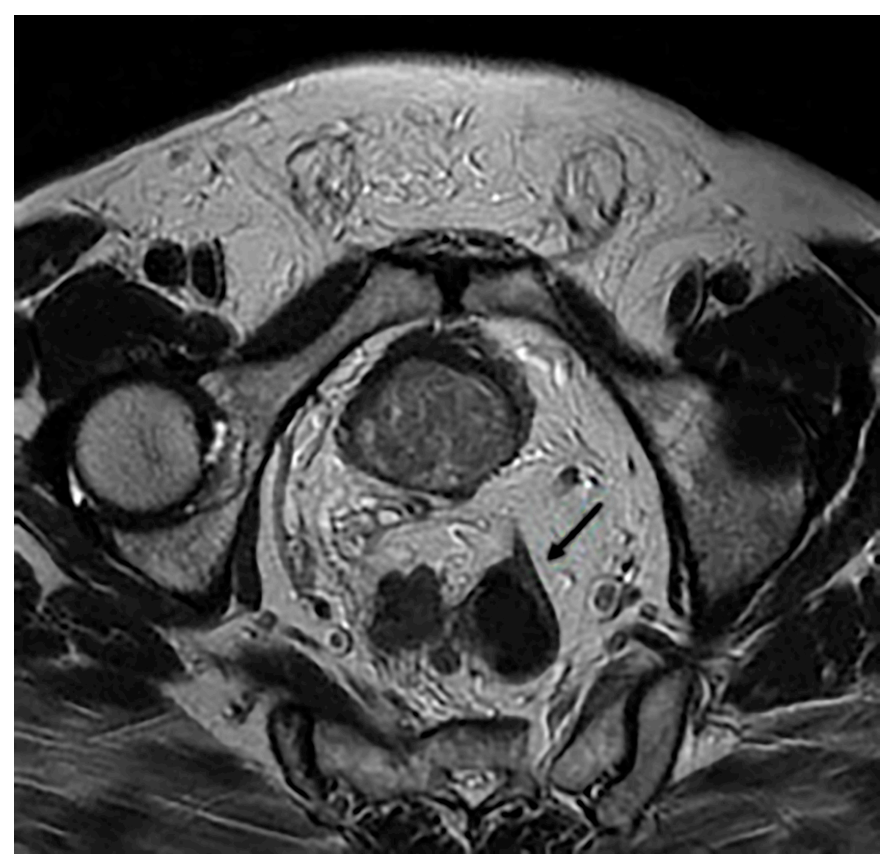

Figure 2: T2W axial section of a patient, who underwent multiparametric prostate MRI, incidentally found neuroendocrine tumor of the rectum (arrow) is apparent.

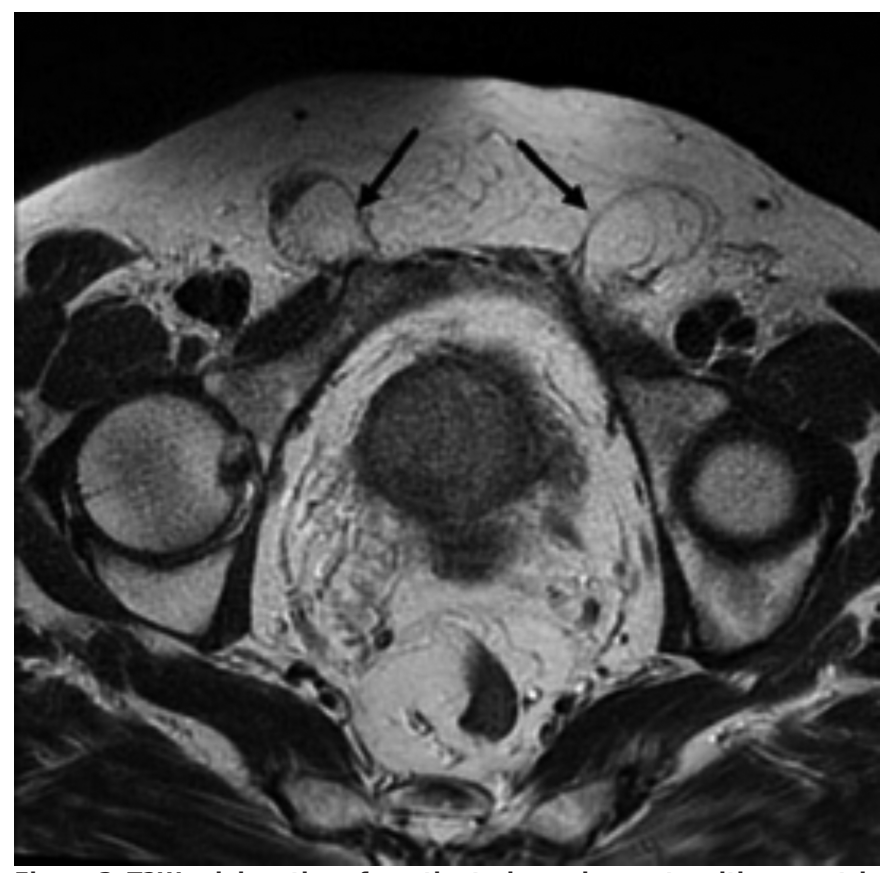

Figure 3: T2W axial section of a patient who underwent multiparametric prostate MRI, reveals incidental bilateral inguinal hernia (arrows).

Prostate biopsy was performed in 90 of 185 patients. Pathology results of 44 patients were reported as benign and 46 patients as malignant. According to the ISUP grading system, 15 (40.5\%) of the patients diagnosed with PCa were grade 1 , of 8 (21.6\%) grade 5 , of $7(18.9 \%)$ grade 3 , of $5(13.5 \%)$ grade 4 , and of 2 (5.4\%) grade $2 \mathrm{PCa}$.

\section{DISCUSSION}

Prostate cancer is the second most common cancer among men, ${ }^{1}$ detecting the disease, limited to the organ, increases the chance of successful treatment and cure. So mp-MRI has played an active role as a diagnostic method in recent years. ${ }^{16}$ Advances in imaging methods contribute to the prevention of unnecessary biopsies. In parallel with these developments, the findings detected incidentally, except for the main investigated disease, are increasing gradually. However, IFs can cause increased cost, patient anxiety, and iatrogenic morbidity.

Table I: Comparison and statistical analysis of IF results of patients according to PI-RADS groups.

\begin{tabular}{|c|c|c|c|}
\hline & $\begin{array}{c}\text { Group } 1 \\
\text { n (\%) }\end{array}$ & $\begin{array}{c}\text { Group } 2 \\
\text { n (\%) }\end{array}$ & p-value \\
\hline Absence IF & $24(52.2)$ & $65(52.4)$ & \multirow{3}{*}{0.970} \\
\hline Benign IF & $21(45.7)$ & $57(46)$ & \\
\hline Malign IF & $1(2.2)$ & $2(1.6)$ & \\
\hline Absence of urologic IF & $31(67.4)$ & $95(76.6)$ & \multirow{2}{*}{0.223} \\
\hline Presence of urologic IF & 15 (32.6) & $29(23.4)$ & \\
\hline Absence of non-urologic IF & $35(76.1)$ & $80(64.5)$ & \multirow{2}{*}{0.152} \\
\hline Presence of non-urologic IF & $11(23.9)$ & $44(35.5)$ & \\
\hline Absence of extraprostatic IF & $24(52.2)$ & $65(52.4)$ & \multirow{4}{*}{0.118} \\
\hline Presence of one extraprostatic IF & 15 (32.6) & $27(21.8)$ & \\
\hline Presence of two extraprostatic IF & $3(6.5)$ & $25(20.2)$ & \\
\hline Presence of three extraprostatic IF & $4(8.7)$ & $7(5.6)$ & \\
\hline Absence of IF in the bladder & $35(76.1)$ & $107(86.3)$ & \multirow{2}{*}{0.111} \\
\hline Presence of IF in the bladder & $11(23.9)$ & $17(13.7)$ & \\
\hline $\begin{array}{l}\text { Absence of benign or malign IF in } \\
\text { the bladder }\end{array}$ & $11(50.0)$ & $42(71.2)$ & \\
\hline $\begin{array}{l}\text { Presence of benign IF in the } \\
\text { bladder }\end{array}$ & $10(45.5)$ & $16(27.1)$ & \multirow{2}{*}{0.192} \\
\hline $\begin{array}{l}\text { Presence of malign IF in the } \\
\text { bladder }\end{array}$ & $1(4.5)$ & $1(1.7)[\mathrm{A} 1]$ & \\
\hline Absence of Hydrocele & $17(77.3)$ & $47(79.7)$ & \multirow{3}{*}{0.665} \\
\hline Presence of unilateral hydrocele & $4(18.2)$ & $7(11.9)$ & \\
\hline Presence of bilateral hydrocele & $1(4.5)$ & $5(8.5)$ & \\
\hline Absence of abdominal free fluid & $22(100)$ & $57(96.6)$ & \multirow{2}{*}{$>0.999$} \\
\hline Presence of abdominal free fluid & $0(0)$ & $2(3.4)$ & \\
\hline Absence of inguinal hernia & $12(54.5)$ & $32(54.2)$ & \multirow{3}{*}{0.397} \\
\hline $\begin{array}{l}\text { Presence of unilateral inguinal } \\
\text { hernia }\end{array}$ & $8(36.4)$ & $15(25.4)$ & \\
\hline $\begin{array}{l}\text { Presence of bilateral inguinal } \\
\text { hernia }\end{array}$ & $2(9.1)$ & $12(20.3)$ & \\
\hline Absence of colorectal IF & $21(95.5)$ & $48(81.4)$ & \multirow{3}{*}{0.278} \\
\hline Presence of sigmoid diverticula & $1(4.5)$ & $10(16.9)$ & \\
\hline Presence of rectal cancer & $0(0)$ & $1(1.7)$ & \\
\hline Absence of Tarlov cyct & $21(95.5)$ & $51(86.4)$ & \multirow{2}{*}{0.432} \\
\hline Presence of Tarlov cyst & $1(4.5)$ & $8(13.6)$ & \\
\hline Absence of lymphadenopathy & $22(100)$ & $56(94.9)$ & \multirow{2}{*}{0.559} \\
\hline Presence of lymphadenopathy & $0(0)$ & $3(5.1)$ & \\
\hline
\end{tabular}

The number of studies focused on this subjectare few in the literature. ${ }^{17-19}$ Therefore, the authors not exactly understand the significance of IFs in mp-MRI. Rachael et al. reported the outcomes of 580 patients and were found 349 IFs in 233 (40\%) patients. Incidental findings were classified in the study as "urologic (PCa related or non-PCa related)" or "non-urologic (high significant or low to moderate significant)". They emphasised that $42 \%$ of mp-MRIs had IFs and only $6.6 \%$ of IFs were clinically significant findings. ${ }^{11}$ The indeterminate liver lesion was 
the most frequent/high significant non-urologic IF and bladder wall thickening/trabeculation was the most common urologic IF according to these results. ${ }^{11}$ In the order of decreasing frequency, suspectedseminal vesicle invasion, suspected osseous metastasis, and pelvic lymphadenopathy suspectedmetastases were reported as PCa-related urologic Ifs. ${ }^{11}$ The study population had a similarage distribution. Categorisation of IFs in mp-MRI that was identified by Yee et al. is an option instead of the three-tier system. ${ }^{20}$ However, the authors did not use this classification in this study and believed that the presence of clinically significant lesions should be individualised. Therefore, it is correct that clinicians make decisions evaluating IFs rather than the interpretation of radiologists. Sometimes an asymptomatic clinically significant IF may be less important to the patient and physician than a symptomatic IF.

Cutaia et al. reported the outcomes of 647 patients and reported 461 IFs in 341 (52.7\%) patients. ${ }^{15}$ Ifs were significantly more common in patients $>65$ years, according to the study results. IFs were classified into related to or not related to the genitourinary system; and subsequently divided into three groups, according to a progressive scale of clinical significance as reported in prior studies. ${ }^{21,22}$ Bladder wall thickening/trabeculation and diverticula were found in $38.8 \%$ of urologic IFs. Colonic diverticulosis was most common IF, not related to the genitourinary system. The present study populations had a similar age distribution and urologic IFs were analogous. The group comparisons in this study, which emphasises the relationship between IF and the advancing age of the patients, show that more care and time should be devoted to the evaluation of elderly patients with imaging methods.

Rayn et al. reported the outcomes of the study, which focused on incidental bladder cancer in $\mathrm{mp}-\mathrm{MRI}, 3,147$ patients were evaluated and $25(0.8 \%)$ bladder lesions were found. Thirteen of 25 patients had stage Ta urothelial carcinoma (UC). Seven of 13 were Ta low-grade and six of 13 were Ta high-grade UC. ${ }^{12}$ In this study, the malignant ep-IFs included two bladder cancer and frequency was $1.08 \%$. Two patients were Ta low-grade. Early detection of UC in mp-MRI may allow early intervention for asymptomatic patients. The absence ofrecurrence and progression for bladder cancer in Rayn etal. and this study, supports the significance of early diagnosis.

Unlike all three studies, the most common IF in mp-MRI was inguinal hernia. The age distribution of inguinal hernia is bimodal with the highest incidence in childhood and after 50 years of age. ${ }^{23}$ The fact that the majority of the patients are over 50 years, may explain the high rate of incidental inguinal hernia detection in mp-MRI.

The frequency of IFs did not differ in terms of all parameters evaluated according to the PI-RADS groups. This may be related to the fact that IFs are mostly benign pathologies. Another possibility may be that incidental lesions do not occur on a genetic background similar to PCa. Although the incidence between groups is not statistically significant, it may be necessary to focus on bladder pathologies for the early diagnosis of bladder cancer.
This study has several limitations that need to be addressed. These include retrospective design, reflecting the data of a single-center, not making comparisons according to age groups, and not having pathological verification. The advanced age of the patients increases the possibility of IFs. To investigate the importance of these findings, first of all, studies with prospective planning, standardised mp-MRI protocol, an expanded number of sections, and pathological confirmation are needed. Despite the limitations mentioned above, this is the first study focusing on the relationship between PI-RADS and IF in $\mathrm{mp}-\mathrm{MRI}$; and the authors think that it contributes to the limited literature knowledge on this topic.

\section{CONCLUSION}

Benign ep-IFs are quite frequent and inguinal hernia is the most common abnormality. The ep-IFs are frequently encountered during $\mathrm{mp}-\mathrm{MRI}$, although rare malignant ep-IFs may be subject to being missed due to focused analysis and interpretation on the prostate. PI-RADS scoring system does not contribute to the diagnosis of IF in mp-MRI. The clinical significance of ep-IFs is not clear currently yet and to be addressed specifically in further studies.

\section{ETHICALAPPROVAL:}

This study was approved by Istanbul Medeniyet Unıversity Goztepe Education and Research Hospital Clinical Trials Ethics Committee. (Ref No. 2018/0481 Date: 12.12.2018).

\section{PATIENTS' CONSENT:}

As this study is a retrospective study, so patients' consent was waived.

\section{CONFLICT OF INTEREST:}

The authors declared no conflict of interest.

\section{AUTHORS' CONTRIBUTION:}

SSE: Conception and design, analysis or interpretation of data for the work, discussion, literature review and critical revision of the manuscript.

NG: Data analysis, results, literature search and discussion.

\section{REFERENCES}

1. Siegel RL, Miller KD, Jemal A, Cancer statistics, 2019. $C A$ Cancer J Clin 2019; 69(1):7-34. doi: 10.3322/caac.21551.

2. Howlader N, Noone A, Krapcho M, Miller D, Bishop K, Kosary C, et al. SEER cancer statistics review, 1975-2014, National cancer institute. Bethesda. Based on November 2016 SEER data submission, posted to the SEER web site, April 2017. 2018.

3. Mohler JL, Antonarakis ES, Armstrong AJ, D'Amico AV, Davis BJ, Dorff T, et al. Prostate cancer, version 2.2019, NCCN clinical practice guidelines in oncology. J Natl Compr Canc Netw 2019; 17(5):479-505. doi: 10.6004/jnccn.2019.0023.

4. Kelly SP, Anderson WF, Rosenberg PS, Cook MB. Past, current, and future incidence rates and burden of metastatic prostate cancer in the United States. Eur Urol Focus 2018; 4(1):121-7. doi: 10.1016/j.euf.2017.10.014.

5. Cui PF, Cong XF, Gao F, Yin JX, Niu ZR, Zhao SC, et al. 
Prognostic factors for overall survival in prostate cancer patients with different site-specific visceral metastases: A study of 1358 patients. World J Clin Cases 2020; 8(1):54-67. doi: 10.12998/wjcc.v8.i1.54.

6. Zhang SG, Wang YH, Ding Y, Wu Y. Long term survival results in advanced prostate cancer treated with combined androgen blockade. Zhonghua Nan Ke Xue 2005; 11(10):770-1, 4.

7. George AK, Turkbey B, Valayil SG, Muthigi A, Mertan F, Kongnyuy $M$, et al. A urologist's perspective on prostate cancer imaging: past, present, and future. Abdom Radiol (NY) 2016; 41(5):805-16. doi: 10.1007/s00261-016-0751-6.

8. Siddiqui MM, Rais-Bahrami S, Turkbey B, George AK, Rothwax J, Shakir N, et al. Comparison of MR/ultrasound fusion-guided biopsy with ultrasound-guided biopsy for the diagnosis of prostate cancer. JAMA 2015; 313(4):390-7. doi: 10.1001/jama.2014.17942.

9. Valerio M, Donaldson I, Emberton M, Ehdaie B, Hadaschik BA, Marks LS, et al. Detection of clinically significant prostate cancer using magnetic resonance imagingultrasound fusion targeted biopsy: A systematic review. Eur Urol 2015; 68(1):8-19. doi: 10.1016/j.eururo.2014.10.026.

10. Quintana L, Ward A, Gerrin SJ, Genega EM, Rosen S, Sanda $M G$, et al. Gleason misclassification rate is independent of number of biopsy cores in systematic biopsy. Urology 2016; 91:143-9. doi: 10.1016/j.urology.2015.12.089.

11. Sherrer RL, Lai WS, Thomas JV, Nix JW, Rais-Bahrami S. Incidental findings on multiparametric MRI performed for evaluation of prostate cancer. Abdom Radiol (NY) 2018; 43(3):696-701. doi: 10.1007/s00261-017-1237-x

12. Rayn KN, Hale GR, Bloom JB, Gold SA, Carvalho FLF, Mehralivand $S$, et al. Incidental bladder cancers found on multiparametric MRI of the prostate gland: A single center experience. Diagn Interv Radiol 2018; 24(5):316-20. doi: 10.5152/dir.2018.18102.

13. Sardari A, Thomas JV, Nix JW, Pietryga JA, Sanyal R, Gordetsky JB, et al. Incidental bladder cancer detected on multiparametric magnetic resonance imaging of the prostate gland. Case Rep Urol 2015; 2015:503154. doi: $10.1155 / 2015 / 503154$.

14. Ho AA, Khara SS, Ferguson DJ, Mohammed MF, Chang SD, Harris AC. A PSA for radiologists: Pictorial review of incidentalomas on prostate magnetic resonance imaging.
Can Assoc Radiol J 2019; 70(2):134-46. doi: 10.1016/j.carj. 2018.09.005.

15. Cutaia G, Tosto G, Cannella R, Bruno A, Leto C, Salvaggio L, et al. Frequency and clinical significance of incidental findings on multiparametric prostate MRI. Radiol Med 2020; 125(2):204-13. doi: 10.1371/journal.pone.0210037.

16. Muthigi A, Sidana A, George AK, Kongnyuy M, Maruf M, Valayil S, et al. Current beliefs and practice patterns among urologists regarding prostate magnetic resonance imaging and magnetic resonance-targeted biopsy. Urol Oncol 2017; 35(1):32 e1- e7. doi: 10.1016/j.urolonc.2016.08.008.

17. Lai WS, Ellenburg J, Lockhart ME, Kolettis PN. Assessing the costs of extraurinary findings of computed tomography urogram in the evaluation of asymptomatic microscopic hematuria. Urol 2016; 95:34-8. doi: 10.1016/j.urology. 2016.06.009.

18. Westbrook JI, Braithwaite J, McIntosh JH. The outcomes for patients with incidental lesions: Serendipitous or iatrogenic? AJR Am J Roentgenol 1998; 171(5):1193-6. doi: 10.2214/ajr.171.5.9798845.

19. Priola AM, Priola SM, Giaj-Levra M, Basso E, Veltri A, Fava C, et al. Clinical implications and added costs of incidental findings in an early detection study of lung cancer by using low-dose spiral computed tomography. Clin Lung Cancer 2013; 14(2):139-48. doi: 10.1016/j.cllc.2012.05.005.

20. Yee J, Kumar NN, Godara S, Casamina JA, Hom R, Galdino $\mathrm{G}$, et al. Extracolonic abnormalities discovered incidentally at CT colonography in a male population. Radiol 2005; 236(2):519-26. doi: 10.1148/radiol.2362040166.

21. Cieszanowski A, Maj E, Kulisiewicz P, Grudzinski IP, Jakoniuk-Glodala K, Chlipala-Nitek I, et al. Non-contrastenhanced whole-body magnetic resonance imaging in the general population: The incidence of abnormal findings in patients 50 years old and younger compared to older subjects. PLoS One 2014; 9(9):e107840. doi: 10.1371/ journal.pone.0107840.

22. Galia M, Albano D, Narese D, Patti C, Chianca V, Di Pietto F, et al. Whole-body MRI in patients with lymphoma: Collateral findings. Radiol Med 2016; 121(10):793-800. doi: 10.1007/ s11547-016-0658-x.

23. Berndsen MR, Gudbjartsson T, Berndsen FH. Inguinal hernia: review. Laeknabladid 2019; 105(9):385-91. doi: 10.17992/lbl.2019.09.247. 